\title{
FACIAL EMOTION DETECTION USING OPEN CV
}

\author{
Yashwanth $\mathbf{D}^{1}$ \\ ${ }^{1}$ Department of Electronics and Communication Engineering \& SVCET college
}

\begin{abstract}
Automatic Face Detection innovations have made numerous upgrades in evolving world. Brilliant ATTENDANCE SYSTEM utilizing ongoing face acknowledgment is a genuine world arrangement which accompanies everyday exercises of taking care of understudies participation. The administration of participation framework can be an extraordinary weight on educators in case it is finished by hands. To determine this issue we utilize auto and brilliant participation framework which is by and large executed with the assistance of biometric called Face Detection. The primary execution steps utilized in this kind of framework are face location and perceiving the identified countenances. Face Detection is an interaction where the framework will actually want to recognize the human faces which will be caught by the camera. Here, we execute a computerized participation the board framework for understudies of the class by utilizing face acknowledgment method..
\end{abstract}

Key Words: Face Detection, Frameworks, Face Recognition, Biometric.

\section{INTRODUCTION ( Size 11, Times New roman)}

You expect representatives to have significant degrees of enthusiastic knowledge while interfacing with clients. Presently, because of advances in Deep Learning, you'll before long anticipate that your software should do the same.Research has shown that more than $90 \%$ of our correspondence can be non-verbal, however innovation has battled to keep up, and conventional code is for the most part terrible at understanding our inflections and expectations. However, feeling acknowledgmentlikewise called Affective Computing - is becoming open to more sorts of engineers. This post will stroll through the intricate details of deciding feeling from information, and a couple of ways you can get some feeling acknowledgment and running yourself.

Facial feeling acknowledgment is the method involved with identifying human feelings from looks. The human mind perceives feelings consequently, and programming has now been fostered that can perceive feelings too. This innovation is turning out to be more exact constantly, and can ultimately peruse feelings just as our minds do. Computer based intelligence can identify feelings by realizing what every look implies and applying that information to the new data introduced to it. Enthusiastic man-made consciousness, or feeling ML, is an innovation that is equipped for perusing, emulating, deciphering, and reacting to human looks and feelings. Feeling Detection Use Cases: TSA Screening, Audience Engagement, And More facial feeling acknowledgment finder

Facial feeling recognition is urgent in day to day existence to distinguish facial feeling or sensation of somebody. We probably won't see that few stages have really taken to recognize human feelings. The current strategy that we follow is we outwardly see the substance of the individual and afterward we distinguish his feelings and afterward we recognize his sentiments. Now and again straightforwardly we can't meet the individual to know his feelings in such case this facial emotion detection is exceptionally useful to know the feeling of a person.So, why not move to a facial feeling location framework which chips away at face acknowledgment strategy? To distinguish the feeling of an individual without any problem

Human looks can be effortlessly characterized into 7 fundamental feelings: cheerful, pitiful, shock, dread, outrage, loathing, and unbiased. Our facial feelings are communicated through enactment of explicit arrangements of facial muscles. These occasionally inconspicuous, yet perplexing, signals in an articulation regularly contain a plentiful measure of data about our perspective. Through facial feeling acknowledgment, we can quantify the impacts that substance and administrations have on the crowd/clients through a simple and minimal expense technique. For instance, retailers might utilize these measurements to assess client interest. Medical care suppliers can offer better support by utilizing extra data about patients' 
passionate state during therapy. Diversion makers can screen crowd commitment in occasions to reliably make wanted substance. People are very much prepared in perusing the feelings of others, indeed, at only 14 months old, infants would already be able to differentiate among glad and tragic. Yet, would computers be able to make a preferable showing over us in getting to enthusiastic states? To respond to the inquiry, We planned a profound learning neural organization that enables machines to make inductions about our enthusiastic states. As such, we give them eyes to perceive what we can see.

\section{Body of Paper}

Facial Expressions can be effectively arranged into 7 essential feelings: neural, disgust, anger, fear, surprise, sad, and happy. Our facial feelings are communicated through initiation of explicit sets of facial muscles. These occasionally inconspicuous, yet mind boggling, signals in an articulation frequently contain a bountiful measure of data about our perspective. Through facial feeling acknowledgment, we can quantify the impacts that substance and administrations have on the crowd/clients through a simple and minimal expense method. For instance, retailers might utilize these measurements to assess client interest. Medical care suppliers can offer better assistance by utilizing extra data about patients' passionate state during treatment. Amusement makers can screen crowd commitment in occasions to reliably make wanted content.Humans are very much prepared in perusing the feelings of others, indeed, at only 14 months old, children can as of now differentiate among cheerful and tragic. However, would computers be able to make a preferred showing over us in getting to passionate states? To respond to the inquiry, We planned a profound learning model that gives machines the capacity to make derivations about our emotional states.

In this paper we will utilize the "HAAR Frontal Face Alt" for distinguishing the appearance of Face in the WebCam. Then, we have the assignment to stack this record, and this can be seen in The label.py program. We run the xml file "classifier=cv2.CascadeClassifier('haarcascade_ frontalface_alt.xml')"

Presently everything can be link with the Label.py Program. So how about we move to the following stage. We will make an picture distinguisher that distinguishes that an individual is dismal, glad, etc and afterward appear this on the OpenCV. This progression will comprise of a few advances: We have to initially make an index titled pictures. In this index, make some 5 or 6 sub indexes with names like neural, disgust, anger, fear, surprise, sad, and happy. You can add more than this.
Now fill these indexes with individual pictures by getting them from the net. E.g., In "Cheerful" registry, fill just those pictures of individual who are glad. Now execute the "facecrop.py" program

Once you have just cleared pictures, you are prepared to retrain . To execute the preparation, go to the main organizer and open CMD/Terminal here and hit the accompanying At last, I've put completely under the "label_image.py" document from where you can get complete information. Presently run the "label.py" program by composing the accompanying in CMD/Terminal. Finally execute python label.py

\section{Haar Cascade Algorithm:}

HAAR Cascade is an AI item location calculation used to distinguish item in a picture or video (model like human appearances) this is generally called as VIOLA JONES algorithmAt first, this wants a ton of +ve pictures of appearances and -ve pictures without appearances to prepare the distinguisher. Then, at that point, we have to extricate highlights from it Object spotting utilizing Haar include based course classifiers is a compelling article recognition technique in their paper, "Fast Object spotting utilizing a Boosted Cascade of Simple Features" in 2001. It is an AI based methodology where a course work is prepared from a ton of +ve and -ve pictures. It is then utilizing to distinguish objects in different pictures. Here we will work with face discovery. At first, the calculation needs a great deal of +ve pictures (pictures of appearances) and -ve (pictures without faces) to prepare the classifier. Then, at that point, we need to separate components from it. For this, haar highlights displayed in beneath picture are utilized. They are actually like our convolutional piece. Each component is a solitary worth got by taking away amount of pixels under white square shape from amount of pixels under dark square shape.
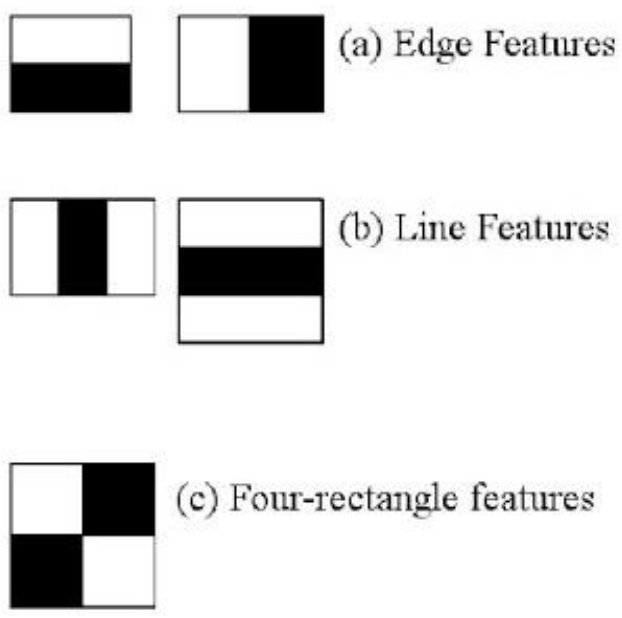
The algorithm has four phases:

1. Haar Feature Selection

2. Making Integral Images

3. Adaboost Training

4. Falling Classifiers

It is notable for having the option to identify faces and body parts in a picture, however can be prepared to distinguish practically any object.Lets take face discovery for instance. At first, the calculation needs a part of positive pictures of countenances and negative pictures without appearances to prepare the classifier. Then we need to separate provisions from it.

\section{Haar Feature Selection}

Initial step is to gather the Haar Features. A Haar include considers neighboring rectangular areas at a particular area in a recognition window, summarizes the pixel forces in every district and computes the distinction between these aggregates.

1. First step is to gather the HAAR Features.

2. It was proposed by ALFERD HAAR in 1909.

HAAR highlights are the important elements for face discovery these resemble rectangular boxes with white and dark locales where white district characterizes splendid region in the face(example: Forehead region, nose connect, teeth, cheeks and so on and dark locales characterizes dull region in a picture like shadows. (model: Hair, eyes, shadows and so on)

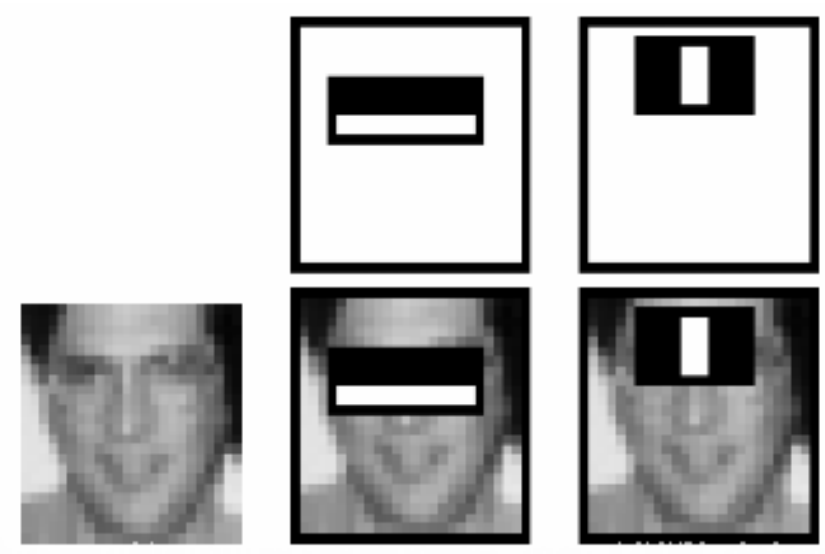

\{Fig: Haar Features)

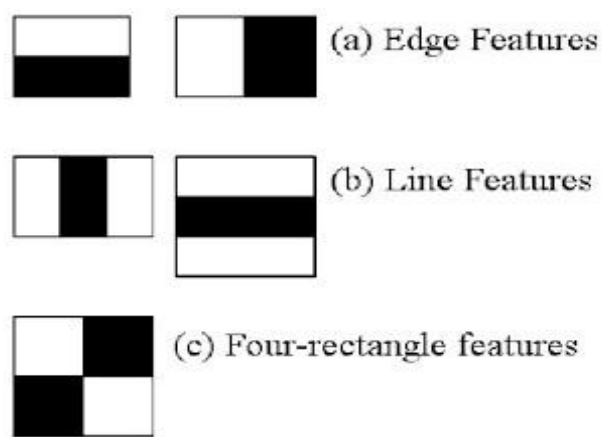

A window of the objective size is moved on the info picture, and for every subside of the picture furthermore, HAAR highlights are determined

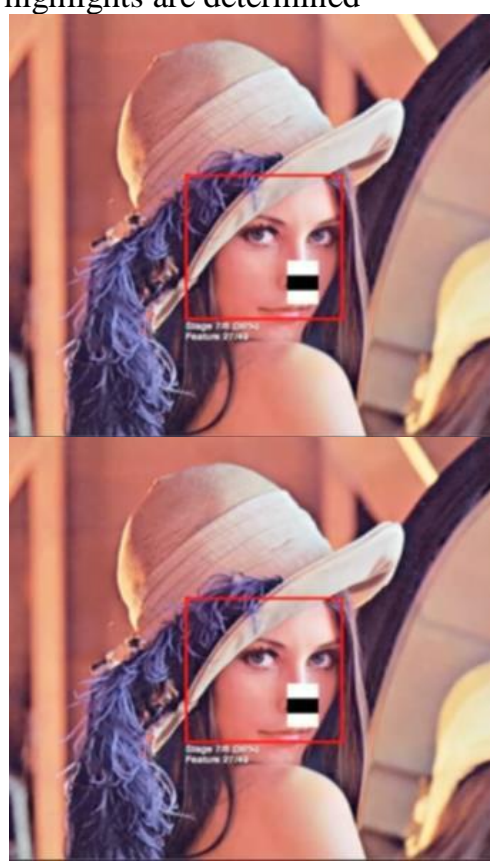

(Haar Features)

summarizes the pixel powers in more contrast districts and computes the distinction between these aggregates.

Face recognition implies recognizing the individual and face check implies confirm the individual who is professed to be (by coordinating with it in certain data set). Its applications are found in different fields like school, universities, associations, industrial facilities, public spots, reconnaissance and so on These strategies are acquiring energy worldwide and reached out with human feeling acknowledgment, its applications are colossal. 


\section{Haar-features}

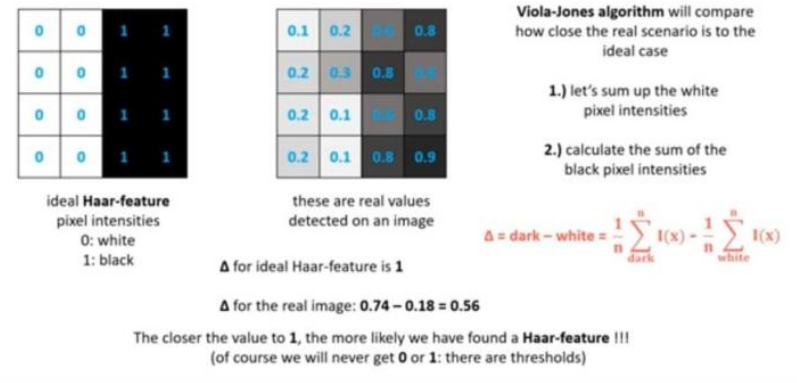

\begin{tabular}{|l|l|l|l|}
\hline 0.1 & 0.2 & 0.6 & 0.8 \\
\hline 0.3 & 0.2 & 0.6 & 0.8 \\
\hline 0.2 & 0.1 & 0.8 & 0.6 \\
\hline 0.2 & 0.1 & 0.8 & 0.9 \\
\hline
\end{tabular}

$\Delta=$ dark - white $=\frac{1}{n} \sum_{\text {dark }}^{n} I(x)-\frac{1}{n} \sum_{\text {white }}^{n} I(x)$

Ideal case : Delta $=(1 / 8) *(8)-(1 / 8) * 0=1$

Real case: Delta $=(1 / 8) *(5.9)-(1 / 8) *(1.3)=0.575$

\section{Integral images:}

Subsequent to finding the haar highlights it summarizes the pixel powers in every district and computes the contrast between these totals. For summarizing the power esteems this idea is utilized. The basic picture is utilized as a fast and viable method of working out the amount of qualities (pixel worth) in a given picture - or a subgroup of a lattice (the given picture).

It can likewise, or is chiefly, utilized for working out the normal power inside a given picture. On the off chance that one needs to utilize the basic picture, it is ordinarily a shrewd plan to ensure the picture is in Greyscalefirst. While making an Integral Image, we need to make a Summed Area Table.
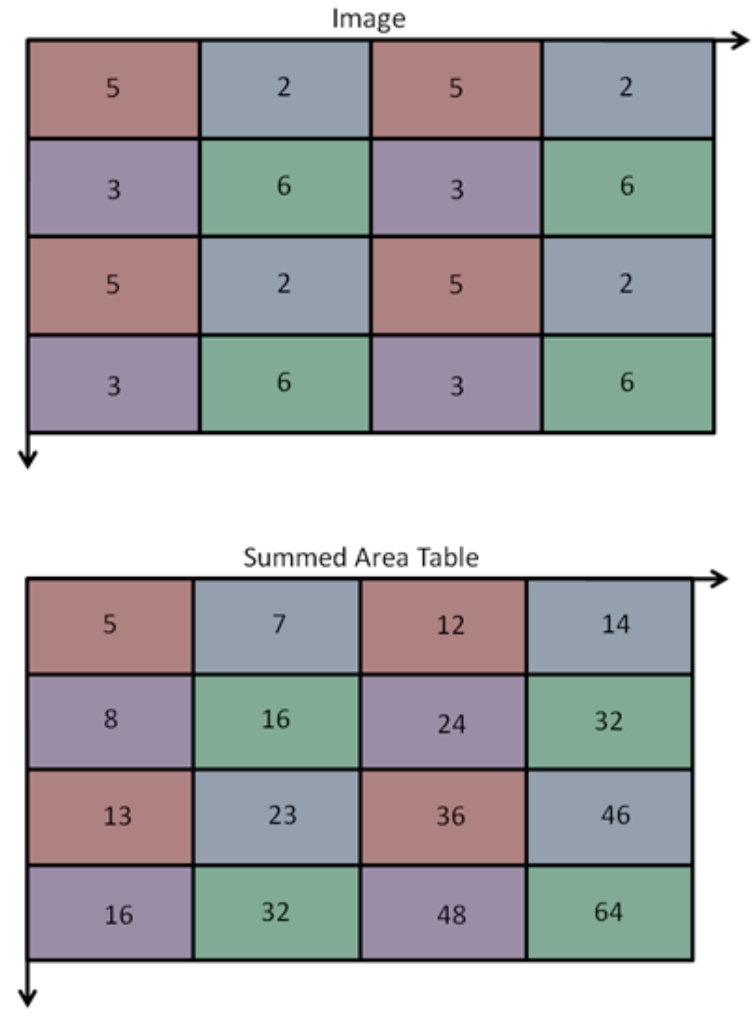

In this table, on the off chance that we go to any point $(x, y)$. The worth is amount of all the pixel esteems above, to the left and obviously including the first pixel worth of $(\mathrm{x}, \mathrm{y})$ itself

\section{Calculating summed area table:}

While making an basic picture we need to make a Sum of Area Table.In the table, in the event that we go to any point ( $\mathrm{x}$ , y). The worth is amount of all the pixel esteems above, to one side and calculating the first pixel worth of $(x, y)$ itself:
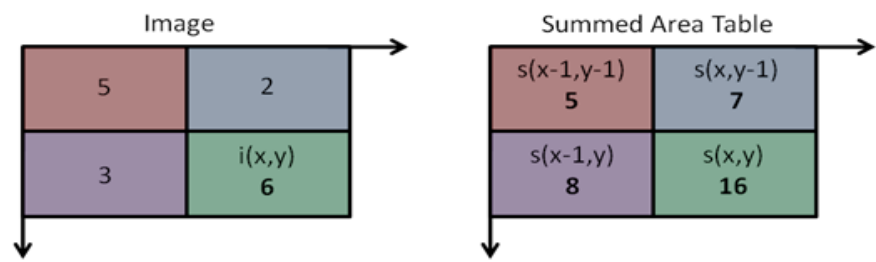

Fig 1.8(calculating summedareatable)

$$
s(x, y)=i(x, y)+s(x-1, y)+s(x, y-1)-s(x-1, y-1)
$$

\section{Calculating an Area in an image:}

Whenever you utilized the condition to ascertain and top off your Sum of the Area Table, the assignment of ascertaining the amount of pixels in some square shape which is a subset of the first picture can be done in steady time. Indeed, believe it or not, in $\mathrm{O}(1)$ intricacy!

To do this we have to utilize 4 qualities from the Sum of the Area Table, that is, 4 exhibit references into the Sum of the Area Table. With these 4 qualities, we then, at that point, add 
or deduct them for the right worth of the amount of the pixels inside that district. To do this, we utilize this condition: Presently, we have produced added region table, this table(integral picture) is helpful to discover normal force of a specific region. suppose we need to ascertain normal power over the space featured in blue

\begin{tabular}{|l|l|l|l|l|}
\hline 5 & 4 & 3 & 8 & 3 \\
\hline 3 & 9 & 1 & 2 & 6 \\
\hline 9 & 6 & 0 & 5 & 7 \\
\hline 7 & 3 & 6 & 5 & 9 \\
\hline 1 & 2 & 2 & 8 & 3 \\
\hline
\end{tabular}

Generally,

$9+1+2+6+0+5+3+6+5=37$

$37 / 9=4.11$

This need a total 9 operations.

Same for 100 operations $100 * 9=900$ operations

But, by using basic picture

\begin{tabular}{|c|c|c|c|c|}
\hline 5 & 9 & 12 & 20 & 23 \\
\hline 8 & 21 & 25 & 35 & 44 \\
\hline 17 & 36 & 40 & 55 & 71 \\
\hline 24 & 46 & 56 & 76 & 101 \\
\hline 25 & 49 & 61 & 89 & 117 \\
\hline
\end{tabular}

Making this integral image required a total of 56 operations. To calculate average intensity of that area, all you have to do is,

$$
\mathbf{i}\left(\mathbf{x}^{\prime}, \mathbf{y}^{\prime}\right)=\mathbf{s}(\mathbf{A})+\mathbf{s}(\mathbf{D})-\mathbf{s}(\mathbf{B})-\mathbf{s}(\mathbf{C})=5+76-20-
$$

$24=37 \quad(37 / 9=4.11)$

\section{Adaboost Algorithm:}

Notwithstanding, among these provisions we determined, the majority of them are unimportant and excess. For a basic $24 * 24$ picture we will have almost $1,60,000$ provisions. So how would we select the best includes out of 160000+ provisions? This is cultivated utilizing an idea called Adaboost which chooses the best provisions and trains the classifiers that utilization them. This calculation develops a "solid" classifier as a direct mix of weighted straightforward "feeble" classifiers. So how would we choose the best provisions out of $160000+$ elements? This is refined utilizing a idea called Adaboost which both chooses the best provisions and trains the dividers that utilization them. This calculation develops a "solid" divider as a direct mix of leaden straightforward "powerless" dividers. The interaction is as per the following.

During the location stage, a window of the objective size is moved over the info picture, and for every subsection of the picture and Haar highlights are determined. You can see this in real life in the video underneath. This distinction is then contrasted with a learned limit that isolates non-objects from objects. Since each Haar highlight is just a "frail divider" (its identification quality is somewhat better than randomguessing) countless Haar highlights are important to portray an object withsufficient exactness and are in this manner coordinated into course dividers to shape a solid divider.

\section{Cascade Classifier}

The cascade classifier contains of an assortment of levels, these level is a troupe of frail students. Each phase of the classifier names the district characterized by the current area of the moving window as one or the other +ve or -ve. +ve shows that an article was found also, -ve demonstrates no articles were found. On the off chance that the name is -ve, the grouping of this area is finished, and the identifier movess the window to the following area. In the event that the name is $+v e$, the classifier passes the area to the following level. The indicator shows an item found at the present window area when the last stage arranges the district as certain. The stages are intended to dismiss -ve examples as quick as could be expected. The supposition that will be simply the greater part of windows don't have the item of interest. On the another hand, genuine up-sides are uncommon also, worth setting aside the effort to confirm.

\section{Results:}

When we execute the program crop.py, we get the output
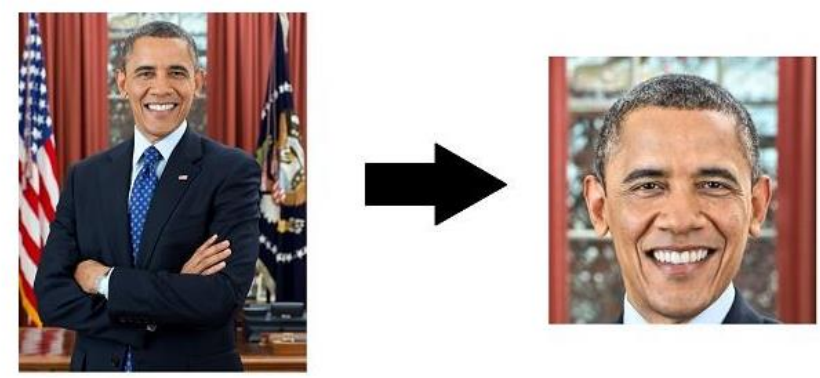

By executing the label.py, the detected face gets an expression like sad, Happy based on the person expression at that time. 


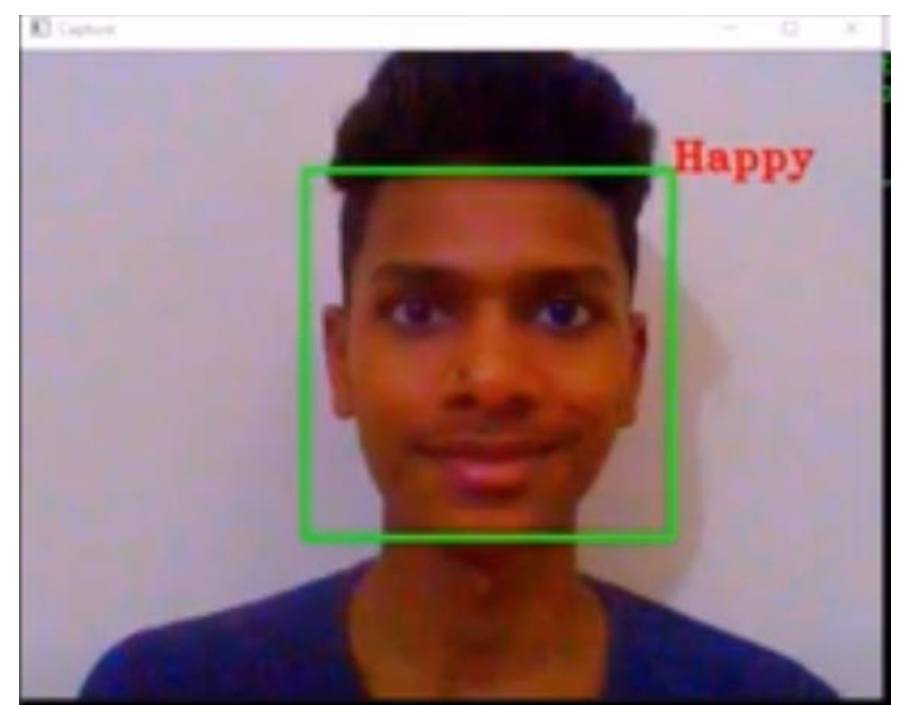

\section{Conclusion:}

This paper proposes a methodology for perceiving the class of facial expressions.Face Identification and Extraction of articulations from facial pictures is helpful in manyapplications, such as mechanical technology vision, video observation, computerized cameras, security andhumanPC communicate particle. This current undertaking's goal was to foster a look acknowledgment framework carrying out the PC dreams and upgrading the advancedfeature extraction and order in face demeanor acknowledgment Look recognitionis an exceptionally difficult issue. More endeavors ought to be made to work on the order execution for significant applications. Our future work will zero in on further developing the execution of the framework and determining more fitting arrangements which may beuseful in numerous genuine applications.

\section{REFERENCES}

[1] M. El Ayaadi, F. Karraeand M. S. Kamal "Survey on emotion recognitions: Features, classification scheme, and database," Pattern Recognit., vol. 44, no. 3, pp. 572-587, 2011.

[2] Y. Lecan, Y. Bengeo, and G. Hintin, "Deep learning," Nature, vol. 521, no. 7553, pp. 436-444, 2015.

[3] J. Schmidhubar, "Deep Learning in neural networks: An overview," Neural Networks, vol. 61, pp. 85-117, 2015.

[4] J. Ngiem, A. Khoslaa, M. Kam, J. Nim, H. Lei, and A. Y. Nig, "Multimodal Deep Learning," Proc. 28th Int. Conf. Mach. Learn., pp. 689-696, 2011.

[5] S. Lugivic M. Harva and I. Dander "Techniques and applications of emotion recognition," 2016 39th Int. Conv. Inf. Commun. Technol. Electron. Microelectron. MIPRO 2016 - Proc., no. November 2017 [16:56, 09/06/2020] hyndavi:

[6] B. Schaller, G. Rigull, and M. Ling, "Emotion recognition combining acoustic features and information in a neural network - belief network architecture," Acoust. Speech, Signal Process., vol. 1, pp. 577-580, 2004

[7] J. Riang, G. Leie, and Y. P. P. Chen, "Acoustic feature selection for automatic emotion recognition from speech," Inf. Process.Manag., vol. 45, no. 3, pp. 315-328, 2009

[8] F. Noruzi, G. Anbarjafaari and N. Akraami "Expressionbased emotion recognition and next reaction prediction," 2017 25th Signal Process. Commun.Appl. Conf. SIU 2017, no. 1, 2017 